\title{
Doping dependence of thermodynamic properties in cuprate superconductors
}

\author{
Huaisong Zhao, Lülin Kuang, and Shiping Feng \\ Department of Physics, Beijing Normal University, Beijing 100875, China
}

\begin{abstract}
The doping and temperature dependence of the thermodynamic properties in cuprate superconductors is studied based on the kinetic energy driven superconducting mechanism. By considering the interplay between the superconducting gap and normal-state pseudogap, the some main features of the doping and temperature dependence of the specific-heat, the condensation energy, and the upper critical field are well reproduced. In particular, it is shown that in analogy to the domelike shape of the doping dependence of the superconducting transition temperature, the maximal upper critical field occurs around the optimal doping, and then decreases in both underdoped and overdoped regimes. Our results also show that the humplike anomaly of the specific-heat near superconducting transition temperature in the underdoped regime can be attributed to the emergence of the normal-state pseudogap in cuprate superconductors.

PACS numbers: 74.25.Bt, 74.20.Mn, 74.20.-z, 74.72.-h
\end{abstract}

\section{INTRODUCTION}

The doping and temperature dependence of the thermodynamic properties for cuprate superconductors has been the subject of much experimental and theoretical investigation ${ }^{1}$. In the conventional superconductors ${ }^{2}$, the absence of the low-energy electron excitations is reflected in the thermodynamic properties, such as the specific heat $C_{\mathrm{v}}$. Although small deviations from exponential behavior have been observed in some conventional superconductors at the low-temperatures, the specific heat of the most conventional superconductors is experimentally found to be exponential at the low-temperatures, since the conventional superconductors are fully gaped 2 . However, the characteristic feature of cuprate superconductors is the existence of four nodes on the Fermi surface ${ }^{3}$, where the d-wave superconducting (SC) gap vanishes $\left.\bar{\Delta}(\mathbf{k})\right|_{\text {at nodes }}=\left.\bar{\Delta}\left(\cos k_{x}-\cos k_{y}\right)\right|_{\text {at nodes }}=0$. In this case, the thermodynamic properties for cuprate superconductors are decreased as some power of the temperature. Moreover, since cuprate superconductors are the doped Mott insulators, obtained by chemically adding charge carriers to a strongly correlated antiferromagnetic insulating state ${ }^{3}$, the thermodynamic properties of cuprate superconductors mainly depend on the extent of dopings, and the regimes have been classified into the underdoped, optimally doped, and overdoped, respectively.

Experimentally, by virtue of systematic studies using the heat capacity measurement technique, some essential features of the evolution of the specific-heat in cuprate superconductors with doping and temperature have been established now $\underline{\underline{4}} \underline{\underline{-8}}$, where the specific-heat in both the SC-state and normal-state in the underdoped regime shows anomalous properties when compared with the case in the optimally doped and overdoped regimes. The early heat capacity measurements $\underline{4} \underline{\underline{6}}$ showed that the specific-heat of cuprate superconductors in the underdoped regime was highly anomalous and deviated strongly from a simple d-wave Bardeen-Cooper-Schrieffer (BCS) form, and the anomalies are a marked reduction in the size of the specific-heat jump near the SC transi- tion temperature $T_{c}$ and a depression in the normal state above $T_{c}$. Later, the heat capacity measurements ${ }^{7}$ indicated that the specific-heat has a humplike anomaly near $T_{c}$ and behaves as a long tail in the underdoped regime, while in the heavily overdoped regime, the anomaly ends sharply just near $T_{c}$. Moreover, it was argued these anomalous specific-heat results as evidence that in the underdoped regime the pseudogap is an intrinsic feature of the normal-state density of states that compets with the SC condensate for the low energy spectral weight 4.8 . Furthermore, by virtue of the magnetization measurement technique, the value of the upper critical field and its doping and temperature dependence have been observed for all the temperature $T \leq T_{c}$ throughout the SC dome $\frac{9-15}{15}$, where at the low temperatures, the upper critical field becomes larger as one moves from the underdoped regime to the optimal doping, and then falls with increasing doping in the overdoped regime, forming a domelike shape doping dependence like $T_{c}$. However, at a given doping concentration, the temperature dependence of the upper critical field follows qualitatively the pair gap temperature dependence ${ }^{9}-15$. Although the doping and temperature dependence of the thermodynamic properties for cuprate superconductors are well-established experimentally $\underline{\underline{4}-15}$ and an agreement has emerged theoretically that the specific-heat of cuprate superconductors in the underdoped regime is not describable within the simple d-wave BCS formalism, its full understanding is still a challenging issue. In particular, the specific-heat of cuprate superconductors has been calculated based on a phenomenological theory of the normal-state pseudogap state $\frac{16}{}$, and the results show that the strong suppression of the specific-heat jump near $T_{c}$ and the corresponding reduction in condensation energy with increased underdoping can be understood as due to the emergence of a pseudogap. However, up to now, the thermodynamic properties of cuprate superconductors have not been treated starting from a microscopic SC theory, and no explicit calculations of the doping and temperature dependence of the upper critical field has been made so far. 
In our recent study ${ }^{17}$, the interplay between the SC gap and normal-state pseudogap in cuprate superconductors is studied based on the kinetic energy driven $\mathrm{SC}$ mechanism 18 , where we show that the interaction between charge carriers and spins directly from the kinetic energy by exchanging spin excitations in the higher power of the doping concentration induces the normalstate pseudogap state in the particle-hole channel and the SC-state in the particle-particle channel, then there is a coexistence of the SC gap and normal-state pseudogap in the whole SC dome. In particular, this normal-state pseudogap is closely related to the quasiparticle coherent weight, and therefore it is a necessary ingredient for superconductivity in cuprate superconductors. Moreover, both the normal-state pseudogap and SC gap are dominated by one energy scale, and they are the result of the strong electron correlation. In this paper, we start from this theoretical framework ${ }^{17}$, and then provide a natural explanation to the doping and temperature dependence of the thermodynamic properties in cuprate superconductors. We evaluate explicitly the specific-heat and upper critical field, and qualitatively reproduced some main features of the heat capacity and magnetization measurements on cuprate superconductors $\stackrel{4-15}{-}$. In particular, we show that in analogy to the domelike shape of the doping dependence of the SC transition temperature, the upper critical field increases with increasing doping in the underdoped regime, and reaches a maximum in the optimal doping, then decreases with increasing doping in the overdoped regime.

The rest of this paper is organized as follows. We present the basic formalism in Section III, and then the quantitative characteristics of the doping and temperature dependence of the thermodynamic properties are discussed in Section [II] where we show that the humplike anomaly of the specific-heat near $T_{c}$ in the underdoped regime can be attributed to the emergence of the normalstate pseudogap in cuprate superconductors. Finally, we give a summary in Section IV

\section{THEORETICAL FRAMEWORK}

In cuprate superconductors, the characteristic feature is the presence of the $\mathrm{CuO}_{2}$ plane ${ }^{3}$. In this case, it is commonly accepted that the essential physics of the doped $\mathrm{CuO}_{2}$ plane $\frac{19}{9}$ is captured by the $t-J$ model on a square lattice,

$$
\begin{aligned}
H & =-t \sum_{l \hat{\eta} \sigma} C_{l \sigma}^{\dagger} C_{l+\hat{\eta} \sigma}+t^{\prime} \sum_{l \hat{\eta}^{\prime} \sigma} C_{l \sigma}^{\dagger} C_{l+\hat{\eta}^{\prime} \sigma}+\mu \sum_{l \sigma} C_{l \sigma}^{\dagger} C_{l \sigma} \\
& +J \sum_{l \hat{\eta}} \mathbf{S}_{l} \cdot \mathbf{S}_{l+\hat{\eta}}
\end{aligned}
$$

where $\hat{\eta}= \pm \hat{x}, \pm \hat{y}, \hat{\eta}^{\prime}= \pm \hat{x} \pm \hat{y}, C_{l \sigma}^{\dagger}\left(C_{l \sigma}\right)$ is the electron creation (annihilation) operator, $\mathbf{S}_{l}=\left(S_{l}^{x}, S_{l}^{y}, S_{l}^{z}\right)$ are spin operators, and $\mu$ is the chemical potential. This $t$ - $J$ model (1) is in the Hilbert subspace with no doubly occupied electron states on the same site, i.e., $\sum_{\sigma} C_{l \sigma}^{\dagger} C_{l \sigma} \leq 1$. To incorporate this electron single occupancy local constraint, the charge-spin separation (CSS) fermion-spin theory 20,21 has been proposed, where the physics of no double occupancy is taken into account by representing the electron as a composite object created by $C_{l \uparrow}=h_{l \uparrow}^{\dagger} S_{l}^{-}$and $C_{l \downarrow}=h_{l \downarrow}^{\dagger} S_{l}^{+}$, with the spinful fermion operator $h_{l \sigma}=e^{-i \Phi_{l \sigma}} h_{l}$ that describes the charge degree of freedom of the electron together with some effects of spin configuration rearrangements due to the presence of the doped hole itself (charge carrier), while the spin operator $S_{l}$ represents the spin degree of freedom of the electron, then the electron single occupancy local constraint is satisfied in analytical calculations. In this CSS fermion-spin representation, the $t$ - $J$ model (11) can be expressed as,

$$
\begin{aligned}
H & =t \sum_{l \hat{\eta}}\left(h_{l+\hat{\eta} \uparrow}^{\dagger} h_{l \uparrow} S_{l}^{+} S_{l+\hat{\eta}}^{-}+h_{l+\hat{\eta} \downarrow}^{\dagger} h_{l \downarrow} S_{l}^{-} S_{l+\hat{\eta}}^{+}\right) \\
& -t^{\prime} \sum_{l \hat{\eta}^{\prime}}\left(h_{l+\hat{\eta}^{\prime} \uparrow}^{\dagger} h_{l \uparrow} S_{l}^{+} S_{l+\hat{\eta}^{\prime}}^{-}+h_{l+\hat{\eta}^{\prime} \downarrow}^{\dagger} h_{l \downarrow} S_{l}^{-} S_{l+\hat{\eta}^{\prime}}^{+}\right) \\
& -\mu \sum_{l \sigma} h_{l \sigma}^{\dagger} h_{l \sigma}+J_{\mathrm{eff}} \sum_{l \hat{\eta}} \mathbf{S}_{l} \cdot \mathbf{S}_{l+\hat{\eta}}
\end{aligned}
$$

where $J_{\text {eff }}=(1-\delta)^{2} J$, and $\delta=\left\langle h_{l \sigma}^{\dagger} h_{l \sigma}\right\rangle=\left\langle h_{l}^{\dagger} h_{l}\right\rangle$ is the charge carrier doping concentration.

For discussions of the doping and temperature dependence of the thermodynamic properties in cuprate superconductors, we need to evaluate the internal energy of the system, which can be separated into two parts in the CSS fermion-spin representation as,

$$
U_{\text {total }}(T, \delta)=U_{\text {charge }}(T, \delta)+U_{\text {spin }}(T, \delta),
$$

with $U_{\text {charge }}(T, \delta)$ and $U_{\text {spin }}(T, \delta)$ are the corresponding contributions from the charge carriers and spins, respectively, and can be expressed as,

$$
\begin{aligned}
U_{\text {charge }}(T, \delta) & =2 \int_{-\infty}^{\infty} \frac{\mathrm{d} \omega}{2 \pi} \omega \rho_{\text {charge }}(\omega, T, \delta) n_{F}(\omega),(4 a) \\
U_{\text {spin }}(T, \delta) & =\int_{-\infty}^{\infty} \frac{\mathrm{d} \omega}{2 \pi} \omega \rho_{\text {spin }}(\omega, T, \delta) n_{B}(\omega)
\end{aligned}
$$

where $\rho_{\text {charge }}(\omega, T, \delta)$ is the charge carrier density of states, $\rho_{\text {spin }}(\omega, T, \delta)$ is the spin density of states, and the two in the charge carrier part of the internal energy is for spin degeneracy, while $n_{F}(\omega)$ and $n_{B}(\omega)$ are the fermion and boson distribution functions, respectively.

As in the conventional superconductors, the key phenomenon occurring in cuprate superconductors in the SC state is the pairing of charge carriers ${ }^{3}$. The system of charge carriers forms pairs of bound charge carriers in the $\mathrm{SC}$ state, while the pairing means that there is an attraction between charge carriers. For a microscopic description of the SC-state of cuprate superconductors, the kinetic energy driven $\mathrm{SC}$ mechanism 18 has been developed 
based on the CSS fermion-spin theory ${ }^{20,21}$, where the attraction between charge carriers mediated by the spin excitations occurs directly through the kinetic energy, then the electron Cooper pairs originating from the charge carrier pairing state are due to the charge-spin recombination, and their condensation reveals the SC ground-state. In particular, the SC transition temperature is identical to the charge carrier pair transition temperature. Within this kinetic energy driven SC mechanism, we have discussed the interplay between the SC-state and normalstate pseudogap state in cuprate superconductors $\frac{17}{}$, and the obtained phase diagram with the two-gap feature is consistent qualitatively with the experimental data observed on different families of cuprate superconductors 22 . Following these previous discussions ${ }^{17,18}$, the full charge carrier diagonal and off-diagonal Green's functions and the mean-field (MF) spin Green's functions can be obtained explicitly as,

$$
\begin{aligned}
g(\mathbf{k}, \omega) & =\frac{U_{1 \mathrm{hk}}^{2}}{\omega-E_{1 \mathrm{~h} \mathbf{k}}}+\frac{V_{1 \mathrm{hk}}^{2}}{\omega+E_{1 \mathrm{~h} \mathbf{k}}} \\
& +\frac{U_{2 \mathrm{~h} \mathbf{k}}^{2}}{\omega-E_{2 \mathrm{hk}}}+\frac{V_{2 \mathrm{~h} \mathbf{k}}^{2}}{\omega+E_{2 \mathrm{~h} \mathbf{k}}}, \\
\Gamma^{\dagger}(\mathbf{k}, \omega) & =-\frac{\alpha_{1 \mathbf{k}} \bar{\Delta}_{\mathrm{h}}(\mathbf{k})}{2 E_{1 \mathrm{hk}}}\left(\frac{1}{\omega-E_{1 \mathrm{~h} \mathbf{k}}}-\frac{1}{\omega+E_{1 \mathrm{~h} \mathbf{k}}}\right) \\
& +\frac{\alpha_{2 \mathbf{k}} \bar{\Delta}_{\mathrm{h}}(\mathbf{k})}{2 E_{2 \mathrm{hk}}}\left(\frac{1}{\omega-E_{2 \mathrm{~h} \mathbf{k}}}-\frac{1}{\omega+E_{2 \mathrm{hk}}}\right), \\
D^{(0)}(\mathbf{p}, \omega) & =\frac{B(\mathbf{p})}{2 \omega(\mathbf{p})}\left(\frac{1}{\omega-\omega(\mathbf{p})}-\frac{1}{\omega+\omega(\mathbf{p})}\right), \\
D_{z}^{(0)}(\mathbf{p}, \omega) & =\frac{B_{z}(\mathbf{p})}{2 \omega_{z}(\mathbf{p})}\left(\frac{1}{\omega-\omega_{z}(\mathbf{p})}-\frac{1}{\omega+\omega_{z}(\mathbf{p})}\right),
\end{aligned}
$$

respectively, where $\alpha_{1 \mathbf{k}}=\left(E_{1 \mathrm{~h} \mathbf{k}}^{2}-M_{\mathbf{k}}^{2}\right) /\left(E_{1 \mathrm{~h} \mathbf{k}}^{2}-E_{2 \mathrm{~h} \mathbf{k}}^{2}\right)$, $\alpha_{2 \mathbf{k}}=\left(E_{2 \mathrm{~h} \mathbf{k}}^{2}-M_{\mathbf{k}}^{2}\right) /\left(E_{1 \mathrm{hk}}^{2}-E_{2 \mathrm{hk}}^{2}\right)$, and there are four coherent charge carrier quasiparticle bands due to the presence of the normal-state pseudogap and SC gap, $E_{1 \mathrm{hk}}$, $-E_{1 \mathrm{hk}}, E_{2 \mathrm{hk}}$, and $-E_{2 \mathrm{hk}}$, with $E_{1 \mathrm{hk}}=\sqrt{\left[\Omega_{\mathbf{k}}+\Theta_{\mathbf{k}}\right] / 2}$, $E_{2 \mathrm{~h} \mathbf{k}}=\sqrt{\left[\Omega_{\mathbf{k}}-\Theta_{\mathbf{k}}\right] / 2}$, and the kernel functions,

$$
\begin{aligned}
& \Omega_{\mathbf{k}}=\xi_{\mathbf{k}}^{2}+M_{\mathbf{k}}^{2}+8 \bar{\Delta}_{\mathrm{pg}}^{2}(\mathbf{k})+\bar{\Delta}_{\mathrm{h}}^{2}(\mathbf{k}) \\
& \Theta_{\mathbf{k}}=\sqrt{\left(\xi_{\mathbf{k}}^{2}-M_{\mathbf{k}}^{2}\right) \beta_{1 \mathbf{k}}+16 \bar{\Delta}_{\mathrm{pg}}^{2}(\mathbf{k}) \beta_{2 \mathbf{k}}+\bar{\Delta}_{\mathrm{h}}^{4}(\mathbf{k})}
\end{aligned}
$$

where $\beta_{1 \mathbf{k}}=\xi_{\mathbf{k}}^{2}-M_{\mathbf{k}}^{2}+2 \bar{\Delta}_{\mathrm{h}}^{2}(\mathbf{k}), \beta_{2 \mathbf{k}}=\left(\xi_{\mathbf{k}}-M_{\mathbf{k}}\right)^{2}+$ $\bar{\Delta}_{\mathrm{h}}^{2}(\mathbf{k})$, the MF charge carrier excitation spectrum $\xi_{\mathbf{k}}=$ $Z t \chi_{1} \gamma_{\mathbf{k}}-Z t^{\prime} \chi_{2} \gamma_{\mathbf{k}}^{\prime}-\mu$, the spin correlation functions $\chi_{1}=$ $\left\langle S_{i}^{+} S_{i+\hat{\eta}}^{-}\right\rangle, \chi_{2}=\left\langle S_{i}^{+} S_{i+\hat{\eta}^{\prime}}^{-}\right\rangle, \gamma_{\mathbf{k}}=(1 / Z) \sum_{\hat{\eta}} e^{i \mathbf{k} \cdot \hat{\eta}}, \gamma_{\mathbf{k}}^{\prime}=$ $(1 / Z) \sum_{\hat{\eta}^{\prime}} e^{i \mathbf{k} \cdot \hat{\eta}^{\prime}}, Z$ is the number of the nearest neighbor or second-nearest neighbor sites, the effective charge carrier d-wave pair gap $\bar{\Delta}_{\mathrm{h}}(\mathbf{k})=\bar{\Delta}_{\mathrm{h}}\left(\cos k_{x}-\cos k_{y}\right) / 2$, and the effective normal-state pseudogap $\bar{\Delta}_{\mathrm{pg}}(\mathbf{k})$ and energy spectrum $M_{\mathrm{k}}$ have been given in Ref. 17, while the co- herence factors,

$$
\begin{aligned}
& U_{1 \mathbf{h k}}^{2}=\frac{1}{2}\left[\alpha_{1 \mathbf{k}}\left(1+\frac{\xi_{\mathbf{k}}}{E_{1 \mathrm{hk}}}\right)-\alpha_{3 \mathbf{k}}\left(1+\frac{M_{\mathbf{k}}}{E_{1 \mathrm{hk}}}\right)\right], \\
& V_{1 \mathrm{hk}}^{2}=\frac{1}{2}\left[\alpha_{1 \mathbf{k}}\left(1-\frac{\xi_{\mathbf{k}}}{E_{1 \mathrm{hk}}}\right)-\alpha_{3 \mathbf{k}}\left(1-\frac{M_{\mathbf{k}}}{E_{1 \mathrm{~h} \mathbf{k}}}\right)\right], \\
& U_{2 \mathbf{h k}}^{2}=-\frac{1}{2}\left[\alpha_{2 \mathbf{k}}\left(1+\frac{\xi_{\mathbf{k}}}{E_{2 \mathrm{hk}}}\right)-\alpha_{3 \mathbf{k}}\left(1+\frac{M_{\mathbf{k}}}{E_{2 \mathrm{hk}}}\right)\right], \\
& V_{2 \mathbf{h k}}^{2}=-\frac{1}{2}\left[\alpha_{2 \mathbf{k}}\left(1-\frac{\xi_{\mathbf{k}}}{E_{2 \mathrm{hk}}}\right)-\alpha_{3 \mathbf{k}}\left(1-\frac{M_{\mathbf{k}}}{E_{2 \mathrm{hk}}}\right)\right],
\end{aligned}
$$

satisfy the sum rule: $U_{1 \mathbf{h k}}^{2}+V_{1 \mathrm{hk}}^{2}+U_{2 \mathrm{hk}}^{2}+V_{2 \mathbf{h k}}^{2}=1$, where $\alpha_{3 \mathbf{k}}=\left[2 \bar{\Delta}_{\mathrm{pg}}(\mathbf{k})\right]^{2} /\left(E_{1 \mathrm{hk}}^{2}-E_{2 \mathrm{~h} \mathbf{k}}^{2}\right)$, while the functions $B(\mathbf{p})$ and $B_{z}(\mathbf{p})$ and the MF spin excitations $\omega(\mathbf{p})$ and $\omega_{z}(\mathbf{p})$ have been given in Refs. 23 and 21 , then all order parameters and chemical potential are determined by the self-consistent calculation.

With the helps of these full charge carrier diagonal Green's function and spin Green's functions in Eq. (5), the charge carrier density of states and spin density of states can be obtained as,

$$
\begin{aligned}
\rho_{\text {charge }}(\omega, T, \delta) & =\frac{1}{N} \sum_{\mathbf{k}} A_{\text {charge }}(\mathbf{k}, \omega, T, \delta), \\
\rho_{\text {spin }}(\omega, T, \delta) & =\frac{1}{2 N} \sum_{\mathbf{k}}\left[A_{\text {spin }}(\mathbf{k}, \omega, T, \delta)\right. \\
& \left.+A_{\text {spin }}^{(z)}(\mathbf{k}, \omega, T, \delta)\right],
\end{aligned}
$$

respectively, where the charge carrier spectral function $A_{\text {charge }}(\mathbf{k}, \omega, T, \delta)=-2 \operatorname{Im} g(\mathbf{k}, \omega)$, and the spin spectral functions $A_{\text {spin }}(\mathbf{k}, \omega, T, \delta)=-2 \operatorname{Im} D^{(0)}(\mathbf{k}, \omega)$ and $A_{\text {spin }}^{(z)}(\mathbf{k}, \omega, T, \delta)=-2 \operatorname{Im} D_{z}^{(0)}(\mathbf{k}, \omega)$. Substituting these corresponding charge carrier density of states and the spin density of states into Eq. (4), and then incorporating the self-consistent equations $\frac{17.23}{}$, we obtain the internal energy of cuprate superconductors in the SC-state as,

$$
\begin{aligned}
U_{\text {total }}^{(s)}(T, \delta) & =-\frac{1}{N} \sum_{\mathbf{k}}\left[E_{1 \mathrm{hk}}\left(\alpha_{1 \mathbf{k}}-\alpha_{3 \mathbf{k}}\right) \operatorname{th}\left(\frac{1}{2} \beta E_{1 \mathbf{h k}}\right)\right. \\
& \left.-E_{2 \mathrm{~h} \mathbf{k}}\left(\alpha_{2 \mathbf{k}}-\alpha_{3 \mathbf{k}}\right) \operatorname{th}\left(\frac{1}{2} \beta E_{2 \mathrm{hk}}\right)\right] \\
& +\frac{1}{N} \sum_{\mathbf{k}} \xi_{\mathbf{k}}+Z J_{\mathrm{eff}}\left(\chi_{1}+\chi_{1}^{z}\right)
\end{aligned}
$$

with the spin correlation function $\chi_{1}^{z}=\left\langle S_{i}^{z} S_{i+\hat{\eta}}^{z}\right\rangle$. In the normal-state, where the charge carrier pair gap $\bar{\Delta}_{\mathrm{h}}=0$, this internal energy is reduced as,

$$
\begin{aligned}
U_{\text {total }}^{(n)}(T, \delta) & =-\frac{1}{N} \sum_{\mathbf{k}}\left[E_{\mathrm{hk}}^{+} \alpha_{1 \mathbf{k}}^{(n)} \operatorname{th}\left(\frac{1}{2} \beta E_{\mathrm{hk}}^{+}\right)\right. \\
& \left.-E_{\mathrm{hk}}^{-} \alpha_{2 \mathbf{k}}^{(n)} \operatorname{th}\left(\frac{1}{2} \beta E_{\mathrm{hk}}^{-}\right)\right] \\
& +\frac{1}{N} \sum_{\mathbf{k}} \xi_{\mathbf{k}}+Z J_{\mathrm{eff}}\left(\chi_{1}+\chi_{1}^{z}\right)
\end{aligned}
$$


with $\alpha_{1 \mathbf{k}}^{(n)}=\left(E_{\mathrm{hk}}^{+}+M_{\mathbf{k}}\right) /\left(E_{\mathrm{hk}}^{+}-E_{\mathrm{hk}}^{-}\right), \quad \alpha_{2 \mathbf{k}}^{(n)}=$ $\left(E_{\mathrm{hk}}^{-}+M_{\mathbf{k}}\right) /\left(E_{\mathrm{hk}}^{+}-E_{\mathrm{hk}}^{-}\right), \quad E_{\mathrm{hk}}^{+}=\left[\xi_{\mathbf{k}}-M_{\mathbf{k}}+\right.$ $\left.\sqrt{\left(\xi_{\mathbf{k}}+M_{\mathbf{k}}\right)^{2}+16 \bar{\Delta}_{\mathrm{pg}}^{2}(\mathbf{k})}\right] / 2$, and $E_{\mathrm{hk}}^{-}=\left[\xi_{\mathbf{k}}-M_{\mathbf{k}}-\right.$ $\left.\sqrt{\left(\xi_{\mathbf{k}}+M_{\mathbf{k}}\right)^{2}+16 \bar{\Delta}_{\mathrm{pg}}^{2}(\mathbf{k})}\right] / 2$.

\section{QUANTITATIVE CHARACTERISTICS OF THE THERMODYNAMIC PROPERTIES}

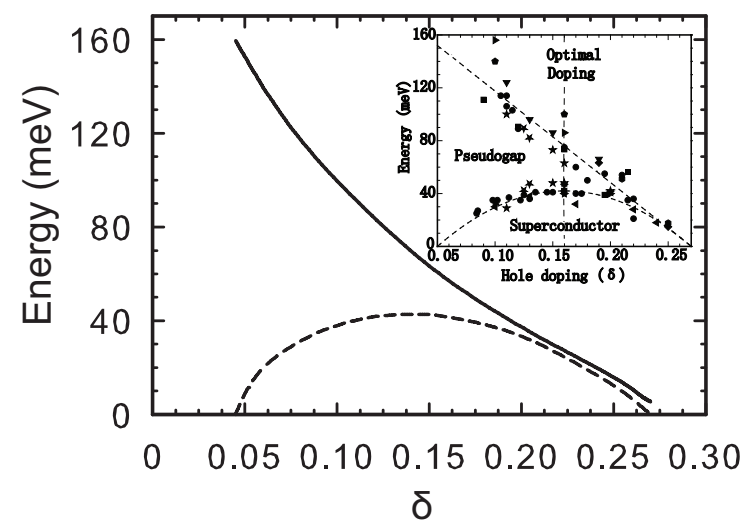

FIG. 1: The magnitude of the effective normal-state pseudogap parameter $\left(2 \bar{\Delta}_{\mathrm{pg}}\right)$ (solid line) and effective charge carrier pair gap parameter $\left(2 \bar{\Delta}_{\mathrm{h}}\right)$ (dashed line) as a function of doping for temperature $T=0.002 J$ with parameters $t / J=2.5$, $t^{\prime} / t=0.3$, and $J=110 \mathrm{meV}$. Inset: the corresponding experimental data of cuprate superconductors taken from Ref. 22.
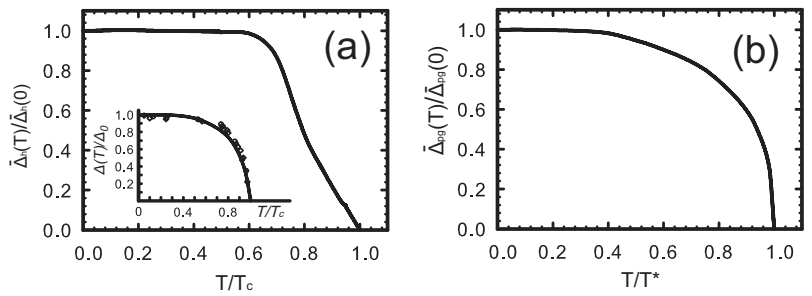

FIG. 2: (a) The effective charge carrier pair gap parameter and (b) the effective normal-state pseudogap parameter as a function of temperature in the doping concentration $\delta=0.09$ with $t / J=2.5, t^{\prime} / t=0.3$, and $J=110 \mathrm{meV}$. Inset in (a): the corresponding experimental data of the pair gap parameter for the underdoped $\mathrm{Bi}_{2} \mathrm{Sr}_{2} \mathrm{Ca}_{2} \mathrm{Cu}_{3} \mathrm{O}_{10+\delta}$ taken from Ref. 24 .

In this section, we discuss some basic behaviors of the doping and temperature dependence of the thermodynamic properties in cuprate superconductors. In cuprate superconductors, although the values of $J, t$, and $t^{\prime}$ are believed to vary somewhat from compound to compound ${ }^{3}$, however, as in our previous studies $\frac{17}{}$, the commonly used parameters in this paper are chosen as $t / J=2.5, t^{\prime} / t=0.3$, and $J=110 \mathrm{meV}$ for a qualitative discussion. In this case, for a complement of the previous analysis of the interplay between the SC gap and normalstate pseudogap in cuprate superconductors 17 , we replot the magnitude of the effective normal-state pseudogap parameter $\left(2 \bar{\Delta}_{\mathrm{pg}}\right)$ (solid line) and effective charge carrier pair gap parameter $\left(2 \bar{\Delta}_{\mathrm{h}}\right)$ (dashed line) as a function of doping for temperature $T=0.002 J$ in Fig. 1 in comparison with the corresponding experimental results 22 observed on different families of cuprate superconductors (inset). In cuprate superconductors, the charge carrier pairing gap parameter measures the strength of the binding of charge carriers into the charge carrier pairs, while the normal-state pseudogap is closely related to the unusual physical properties. Our theoretical results in Fig. 1 reproduce qualitatively the two-gap feature observed on cuprate superconductors 22 , and show that the effective charge carrier pair gap parameter increases with increasing doping in the underdoped regime, and reaches a maximum in the optimal doping, then decreases with increasing doping in the overdoped regime $\frac{18,23}{}$. However, in contrast to the case of the effective charge carrier pair gap parameter in the underdoped regime, the magnitude of the effective normal-state pseudogap parameter smoothly increases with decreasing doping in the underdoped regime, this leads to that the magnitude of the effective normal-state pseudogap parameter is much larger than the effective charge carrier pair gap parameter in the underdoped regime. Moreover, the magnitude of the normal-state pseudogap parameter seems to merge with the charge carrier pair gap parameter in the overdoped regime, eventually disappearing together with superconductivity at the doping concentrations larger than the doping concentration $\delta \sim 0.27$. Furthermore, these effective charge carrier pair gap parameter and effective normal-state pseudogap parameter are strongly temperature dependent. To show this point clearly, we plot (a) the effective charge carrier pair gap parameter and (b) the effective normal-state pseudogap parameter as a function of temperature at the doping concentration $\delta=0.09$ in Fig. 2 For comparison, the corresponding experimental result of the pair gap parameter ${ }^{24}$ for the underdoped $\mathrm{Bi}_{2} \mathrm{Sr}_{2} \mathrm{Ca}_{2} \mathrm{Cu}_{3} \mathrm{O}_{10+\delta}$ is also shown in Fig. 2 [inset in (a)]. Obviously, both the effective charge carrier pair gap parameter and the effective normal-state pseudogap parameter have a similar temperature dependence, and they decreases with increasing temperatures, however, the effective charge carrier pair gap parameter vanishes at $T_{c}$, while the effective normal-state pseudogap parameter vanishes at the normal-state pseudogap crossover temperature $T^{*}$, where $T^{*}$ is much larger than $T_{c}$ in the underdoped regime $\frac{17}{}$.

\section{A. Doping and temperature dependence of the specific-heat}

In the following discussions, we discuss the doping dependence of the specific-heat in cuprate superconductors. With the helps of Eqs. (9) and (10), the specific-heat can 

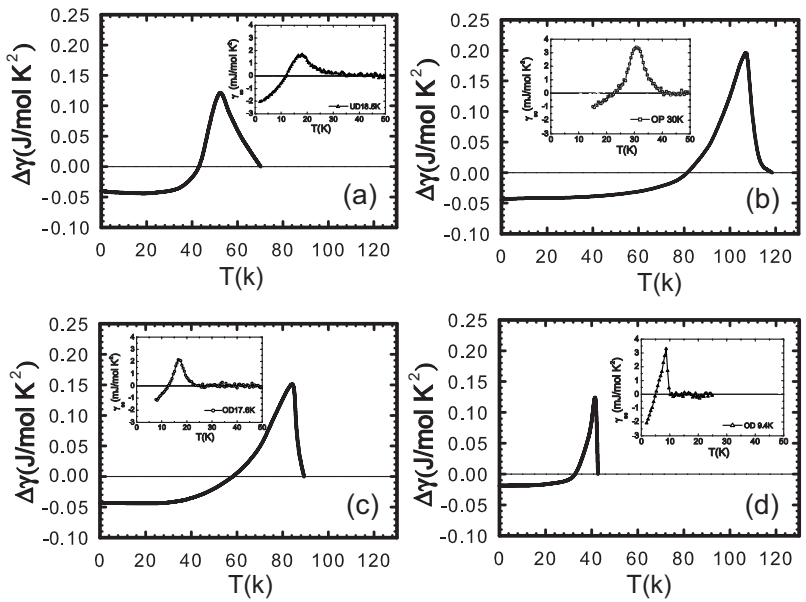

FIG. 3: The specific-heat coefficient as a function of temperature at (a) $\delta=0.09$, (b) $\delta=0.15$, (c) $\delta=0.18$, and (d) $\delta=0.25$ with $t / J=2.5, t^{\prime} / t=0.3$, and $J=110 \mathrm{meV}$. Insets: the corresponding experimental data of $\mathrm{Bi}_{2} \mathrm{Sr}_{2-x} \mathrm{La}_{x} \mathrm{CuO}_{6+\delta}$ taken from Ref. 7.

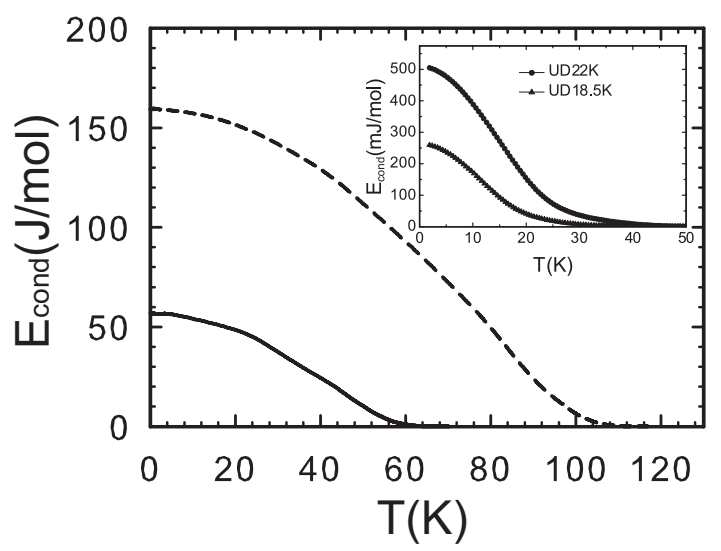

FIG. 4: The condensation energy as a function of temperature at $\delta=0.09$ (solid line) and $\delta=0.15$ (dashed line) with $t / J=$ $2.5, t^{\prime} / t=0.3$, and $J=110 \mathrm{meV}$. Insets: the corresponding experimental data for $\mathrm{Bi}_{2} \mathrm{Sr}_{2-x} \mathrm{La}_{x} \mathrm{CuO}_{6+\delta}$ in the underdoped regime taken from Ref. 7 .

be obtained by evaluating the temperature-derivative of the internal energies as,

$$
C_{\mathrm{v}}^{(a)}(T, \delta)=\frac{\mathrm{d} U^{(a)}(T, \delta)}{\mathrm{d} T}=\gamma_{a}(T, \delta) T,
$$

where $a=s, n$, and $\gamma_{a}(T, \delta)$ is the doping and temperature dependence of the specific-heat coefficient. In this case, we have performed a calculation for the specificheat coefficient, and the results of $\Delta \gamma(T, \delta)=\gamma_{s}(T, \delta)-$ $\gamma_{n}(T, \delta)$ as a function of temperature in the underdoping (a) $\delta=0.09$, (b) the optimal doping $\delta=0.15$, (c) the overdoping $\delta=0.18$, and (d) the heavily overdoping $\delta=0.25$ are plotted in Fig. 3 in comparison with the corresponding experimental data 7 for $\mathrm{Bi}_{2} \mathrm{Sr}_{2-x} \mathrm{La}_{x} \mathrm{CuO}_{6+\delta}$ (inset). It is shown clearly that our present theoretical results capture all essential qualitative features of the doping dependence of the specific-heat observed experimentally on cuprate superconductors ${ }^{4-8}$. In the underdoped regime, the specific-heat jump near $T_{c}$ is strongly suppressed, therefore there is no steplike specific-heat anomaly near $T_{c}$, instead, it shows a humplike peak and remains as long tail of $\gamma_{s}(T, \delta)$. However, in the optimal doping, although the specific-heat anomaly is still not a sharp steplike, it shows a symmetric peak, and therefore there is a tendency towards to the steplike specific-heat anomaly with increasing doping. This tendency is particularly obvious in the overdoped regime, where the long tail appeared in the underdoped regime becomes much shorter, then the specific-heat anomaly ends near $T_{c}$ in the heavily overdoped regime, and a steplike BCS transition with the absence of the long tail appears.

\section{B. Doping and temperature dependence of the condensation energy}

For a superconductor, it undergoes a transition from the normal-state to the SC-state because this transition can lower the total free energy, and then the energy difference between the normal-state $F^{(n)}(T, \delta)$, extrapolated to zero temperature, and the SC-state $F^{(s)}(T, \delta)$, is defined as the condensation energy $E_{\text {cond }}(T, \delta)$,

$$
E_{\text {cond }}(T, \delta)=F^{(n)}(T, \delta)-F^{(s)}(T, \delta),
$$

where the free energies are obtained in terms of the corresponding internal energies in Eqs. (9) and (10) as,

$$
F^{(a)}(T, \delta)=U^{(a)}(T, \delta)-T S^{(a)}(T, \delta),
$$

with the related entropy of the system is evaluated from the specific-heat coefficient in Eq. (11) as,

$$
S^{(a)}(T, \delta)=\int_{0}^{T} \gamma_{a}\left(T^{\prime}, \delta\right) \mathrm{d} T^{\prime} .
$$

Alternatively, this condensation energy can also be obtained by integrating the difference in the specific-heat coefficients in Eq. (11) in the normal-state and the SCstate $^{7}$ from zero temperature to $T_{c}$. In this cae, we plot the condensation energy $E_{\text {cond }}(T, \delta)$ as a function of temperature at $\delta=0.09$ (solid line) and $\delta=0.15$ (dashed line) in Fig. 4 in comparison with the corresponding experimental data 7 for $\mathrm{Bi}_{2} \mathrm{Sr}_{2-x} \mathrm{La}_{x} \mathrm{CuO}_{6+\delta}$ in the underdoped regime (inset). Our results show that in the underdoped regime, the condensation energy increases with increasing doping, then it follows qualitatively a pair gap type temperature dependence, and disappears at $T_{c}$, in qualitative agreement with experimental data ${ }^{4}-8$.

\section{Doping and temperature dependence of the upper critical field}

Now we turn to discuss the doping and temperature dependence of the upper critical field $B_{c}(T, \delta)$. For a 


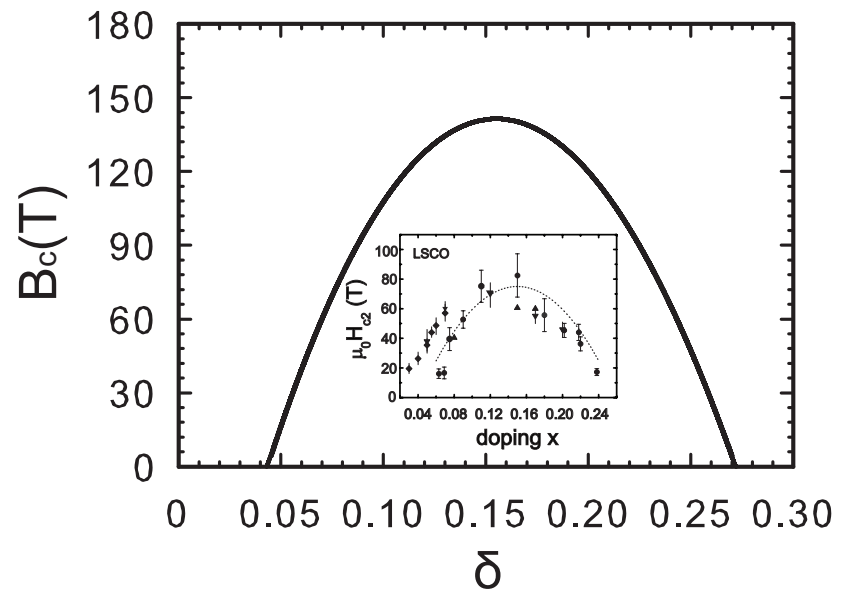

FIG. 5: Doping dependence of the upper critical field with $T=0.002 J$ for $t / J=2.5, t^{\prime} / t=0.3$, and $J=110 \mathrm{meV}$. Insets: the experimental data for $\mathrm{La}_{2-x} \mathrm{Sr}_{x} \mathrm{CuO}_{4}$ taken from Ref. 15.

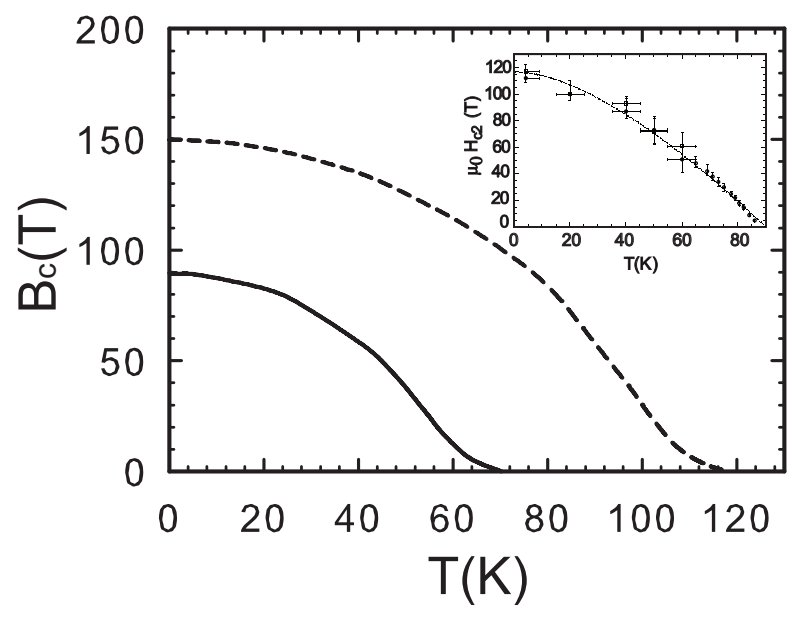

FIG. 6: Temperature dependence of the upper critical field at $\delta=0.09$ (solid line) and $\delta=0.15$ (dashed line) for $t / J=2.5$, $t^{\prime} / t=0.3$, and $J=110 \mathrm{meV}$. Inset: the corresponding experimental result for the slightly underdoped $\mathrm{YBa}_{2} \mathrm{Cu}_{3} \mathrm{O}_{7-\delta}$ taken from Ref. 13 .

given doping concentration, the upper critical field is defined as the critical field that destroys the SC-state at the zero temperature, therefore the upper critical field also measures the strength of the binding of charge carriers into charge carrier pairs like the pair gap parameter. This doping and temperature dependence of the upper critical field is closely related to the doping and temperature dependence of the condensation energy (12), and can be obtained as,

$$
\frac{1}{2 \mu_{0}} B_{c}^{2}(T, \delta)=E_{\text {cond }}(T, \delta) \text {. }
$$

In this case, we have performed firstly a calculation for the doping dependence of the upper critical field $B_{c}(T, \delta)$ at the low temperatures, and the result of $B_{c}(T, \delta)$ as a function of doping with temperature $T=0.002 \mathrm{~J}$ is plotted in Fig. 5. For comparison, the corresponding experimental result ${ }^{15}$ for $\mathrm{La}_{2-x} \mathrm{Sr}_{x} \mathrm{CuO}_{4}$ is also shown in Fig. 5 (inset). Obviously, in analogy to the domelike shape of the doping dependence of $T_{c}$ and pair gap parameter, the upper critical field increases with increasing doping in the underdoped regime, and reaches a maximum in the optimal doping, then decreases with increasing doping in the overdoped regime. This domelike shape of the doping dependence of the upper critical field is well consistent with the experimental data ${ }^{9-15}$. Furthermore, we have discussed the temperature dependence of the upper critical field, and the results of $B_{c}(T, \delta)$ as a function of temperature at $\delta=0.09$ (solid line) and $\delta=0.15$ (dashed line) are plotted in Fig. 6 in comparison with the corresponding experimental result 13 of the slightly underdoped $\mathrm{YBa}_{2} \mathrm{Cu}_{3} \mathrm{O}_{7-\delta}$ (inset). Our results indicate that as in the case of the temperature dependence of the condensation energy shown in Fig. 4, the upper critical field $B_{c}(T)$ also follows qualitatively the pair gap type temperature dependence, i.e., it decreases with increasing temperature, and vanishes at $T_{c}$, which is also qualitatively consistent with the experimental results ${ }^{9}-15$. Since the upper critical field $B_{c}(T, \delta)$ (then the condensation energy) is closely related to the difference between the free energies in the SC-state and normal-state, the charge carrier pair gap parameter is relevant as shown in Eqs. (15) and (13), i.e., the variation of the upper critical field (then the condensation energy) with doping and temperature is coupled to the doping and temperature dependence of the charge carrier pair gap parameter $\bar{\Delta}_{\mathrm{h}}$ in cuprate superconductors. In this case, our present results of the upper critical field and its domelike shape of the doping dependence and pair gap type temperature dependence also are a natural consequence of the results for the charge carrier pair gap parameter and its domelike shape of the doping dependence and similar BCS type temperature dependence in the framework of the kinetic energy driven SC mechanism ${ }^{18}$ as shown in Fig. 1 and Fig. 2.

The doping and temperature dependence of the coherence length $\zeta(T, \delta)$ is one of the important characteristic parameters of cuprate superconductors. Although it can not be measured directly, it is closely related to the doping and temperature dependence of the upper critical field as $\zeta^{2}(T, \delta)=\Phi_{0} / 2 \pi B_{c}(T, \delta)$, where $\Phi_{0}=h c / 2 e$ is the magnetic flux quantum. In this case, with the help of the doping and temperature dependence of the upper critical field in Eq. (15), we can obtain the doping and temperature dependence of the coherence length $\zeta(T, \delta)$, and the results of $\zeta(T, \delta)$ as a function of doping with temperature $T=0.002 \mathrm{~J}$ are plotted in Fig. 7 in comparison with the corresponding experimental data ${ }^{15}$ of $\mathrm{La}_{2-x} \mathrm{Sr}_{x} \mathrm{CuO}_{4}$ (inset). Obviously, the main feature of the doping dependence of the coherence length $\zeta(T, \delta)$ obtained from the experiments 15 is reproduced, where in contrast to the case of the doping dependence of the upper critical field, the coherence length $\zeta(T, \delta)$ in cuprate 
superconductors reaches a minimum around the optimal doping, then grows in both the underdoped and overdoped regimes.

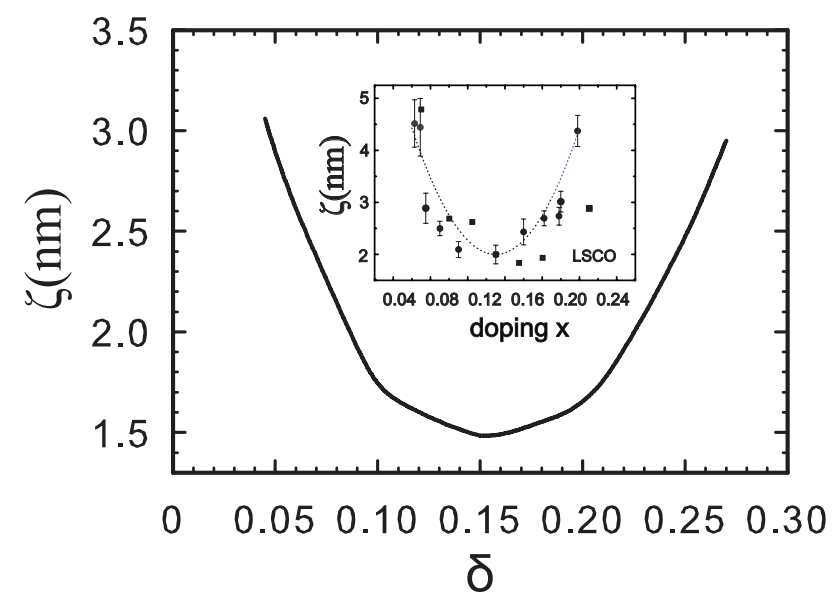

FIG. 7: The coherence length as a function of doping in $T=$ $0.002 J$ for $t / J=2.5, t^{\prime} / t=0.3$, and $J=110 \mathrm{meV}$. Inset: the experimental results for $\mathrm{La}_{2-x} \mathrm{Sr}_{x} \mathrm{CuO}_{4}$ taken from Ref. 15.

The essential physics of the humplike anomaly of the specific-heat near $T_{c}$ in cuprate superconductors in the underdoped regime can be attributed to the emergence of the normal-state pseudogap 17 . This follows a fact that in the framework of the kinetic energy driven SC mechanism $\frac{18}{}$, the normal-state pseudogap state is particularly obvious in the underdoped regime as shown in Fig. [1 i.e., the magnitude of the normal-state pseudogap is much larger than that of the pair gap in the underdoped regime, then it smoothly decreases upon increasing doping as mentioned above. In this case, the sharp peak in the charge carrier density of states in the absence of the normal-state pseudogap is spread out due to the band split in the presence of the normal-state pseudogap, reflecting a suppression for the strength of the charge carrier density of states. In particular, this suppression for the strength of the charge carrier density of states follows the same doping dependent behavior of the normal-state pseudogap, i.e., it decreases with increasing doping. This strong suppression for the strength of the charge carrier density of states in the underdoped regime leads to a strong suppression of the specific-heat jump near $T_{c}$, then the humplike anomaly near $T_{c}$ with a long tail in the underdoped regime is a natural consequence of the spread of the charge carrier density of states. However, the range of this long tail decreases with increasing doping as the suppression for the strength of the charge carrier density of states decreases upon increasing doping. In particular, in the heavily overdoped regime, the normal-state pseudogap merges with the charge carrier pair gap parameter as shown in Fig. 11 This reflects a fact that in the heavily overdoped regime, when the temperature $T=T_{c}$, the charge carrier pair gap parameter $\bar{\Delta}_{\mathrm{h}}=0$, and at the same time, the normal-state pseudogap is negligible, i.e., $\bar{\Delta}_{\mathrm{pg}} \approx 0$, this leads to a disappearance of the suppression for the strength of the charge carrier density of states near $T_{c}$. In this case, the full charge carrier diagonal and off-diagonal Green's functions (5) near $T_{c}$ can be induced as a simple d-wave BCS formalism 18,23 ,

$$
\begin{aligned}
g(\mathbf{k}, \omega) & =\frac{U_{\mathrm{hk}}^{2}}{\omega-E_{\mathrm{hk}}}+\frac{V_{\mathrm{hk}}^{2}}{\omega+E_{\mathrm{hk}}}, \\
\Gamma^{\dagger}(\mathbf{k}, \omega) & =-\frac{\bar{\Delta}_{\mathrm{h}}(\mathbf{k})}{2 E_{\mathrm{hk}}}\left(\frac{1}{\omega-E_{\mathrm{hk}}}-\frac{1}{\omega+E_{\mathrm{hk}}}\right),
\end{aligned}
$$

although the pairing mechanism is driven by the kinetic energy by exchanging spin excitations, where the charge carrier qasiparticle coherence factors $U_{\mathrm{hk}}^{2}=(1+$ $\left.\xi_{\mathbf{k}} / E_{\mathrm{hk}}\right) / 2$ and $V_{\mathrm{hk}}^{2}=\left(1-\xi_{\mathbf{k}} / E_{\mathrm{hk}}\right) / 2$, and the charge carrier quasiparticle spectrum $E_{\mathrm{hk}}=\sqrt{\xi_{\mathbf{k}}^{2}+\left|\bar{\Delta}_{\mathrm{h}}(\mathbf{k})\right|^{2}}$. This simple d-wave BCS formalism (16) leads to that the specific-heat anomaly ends sharply just near $T_{c}$. This is also why the humplike anomaly near $T_{c}$ with a long tail of the specific-heat appeared obviously in the underdoped regime is absent in the heavily overdoped regime.

\section{CONCLUSIONS}

Based on the $t-J$ model, we have discussed the doping and temperature dependence of the thermodynamic properties in cuprate superconductors. By considering the interplay between the SC gap and normal-state pseudogap within the framework of the kinetic energy driven SC mechanism, we have reproduced qualitatively some main features of the doping and temperature dependence of the specific-heat, the condensation energy, and the upper critical field. The specific-heat shows a humplike peak and remains as long tail in the underdoped regime, however, this long tail is absent in the heavily overdoped regime, and then the specific-heat shows a steplike BCS transition, while the condensation energy increases with increasing doping in the underdoped regime, and follows a pair gap type temperature dependence. Moreover, in analogy to the domelike shape of the doping dependence of $T_{c}$, the maximal upper critical field occurs around the optimal doping, and then decreases in both underdoped and overdoped regimes. Our results also show that the striking behavior of the specific-heat humplike anomaly near $T_{c}$ is closely related to the doping and temperature dependence of the normal-state pseudogap. Since the knowledge of the doping and temperature dependence of the thermodynamic properties in cuprate superconductors is of considerable importance as a test for theories of the normal-state and SC-state, the qualitative agreement between the present theoretical results and experimental data also provides an important confirmation of the nature of the $\mathrm{SC}$ phase of cuprate superconductors as a coexistence of the d-wave SC-state and normal-state pseudogap state in the whole SC dome within the kinetic energy driven SC mechanism. 


\section{Acknowledgments}

The authors would like to thank Dr. Zheyu Huang for helpful discussions. This work was supported by the funds from the Ministry of Science and Technology of China under Grant Nos. 2011CB921700 and 2012CB821403, and the National Natural Science Foundation of China under Grant No. 11074023.
1 See, e.g., the review, A. Junod, in Physical Properties of High Temperature Superconductors II, edited by D. M. Ginsberg (World Scientific, Singapore, 1990), p. 13.

2 See, e.g., the review, J. R. Schrieffer, Theory of Superconductivity (Addison-Wesley, San Francisco, 1964).

3 See, e.g., the review, A. Damascelli, Z. Hussain, and Z.-X. Shen, Rev. Mod. Phys. 75 (2003) 473.

4 J. W. Loram, K. A. Mirza, J. R. Cooper, W. Y. Liang, J. M. Wade, J. Supercond. 7 (1994) 243; J. W. Loram, K. A. Mirza, J. M. Wade, J. R. Cooper, W. Y. Liang, Physica C 235-240 (1994) 134; J. W. Loram, K. A. Mirza, J. R. Cooper, J. L. Tallon, Physica C 282-287 (1997) 1405.

${ }^{5}$ Kathryn A. Moler, David L. Sisson, Jeffrey S. Urbach, Malcolm R. Beasley, Aharon Kapitulnik, David J. Baar, Ruixing Liang, and Walter N. Hardy, Phys. Rev. B. 55 (1997) 3954.

6 Alain Junod, Andreas Erb, and Christophe Renner, Physica C 317-318 (1999) 333; Yuxing Wang, Alain Junod, Bernard Revaz, and Andreas Erb, Physica C 341-348 (2000) 1073.

7 Hai-Hu Wen, Gang Mu, Huiqian Luo, Huan Yang, Lei Shan, Cong Ren, Peng Cheng, Jing Yan, and Lei Fang, Phys. Rev. Lett. 103 (2009) 067002.

8 W. Y. Liang, J. W. Loram, K. A. Mirza, N. Athanassopoulou, J. R. Cooper, Physica C 263 (1996) 277; J. W. Loram, J. L. Luo, J. R. Cooper, W. Y. Liang, J. L. Tallon, Physica C 341-348 (2000) 831.

9 S. L. Lee, P. Zimmermann, H. Keller, M. Warden, I. M. Savić, R. Schauwecker, D. Zech, R. Cubitt, E. M. Forgan, P. H. Kes, T. W. Li, A. A. Menovsky, and Z. Tarnawski, Phys. Rev. Lett. 71 (1993) 3862.

10 M. Nideröst, R. Frassanito, M. Saalfrank, A. C. Mota, G. Blatter, V. N. Zavaritsky, T. W. Li, and P. H. Kes, Phys.
Rev. Lett. 81 (1998) 3231.

11 H. Nakagawa, N. Miura, and Y. Enomoto, J. Phys.: Condens. Matter 10 (1998) 11571.

12 J. L. Luo, J. W. Loram, J. R. Cooper, J. Tallon, Physica C 341-348 (2000) 1837.

13 N. Miura, H. Nakagawa, T. Sekitani, M. Naito, H. Sato, and Y. Enomoto, Physica B 319 (2000) 310.

14 Yayu Wang, Lu Li, and N. P. Ong, Phys. Rev. B. 73 (2006) 024510.

15 Y. Wang and H.-H. Wen, Europhys. Lett. 81 (2008) 57007.

16 Zlatko Teŝanović, Nature Phys. 4 (2008) 408.

17 Shiping Feng, Huaisong Zhao, and Zheyu Huang, Phys. Rev. B. 85 (2012) 054509.

18 Shiping Feng, Phys. Rev. B 68 (2003) 184501; Shiping Feng, Tianxing Ma, and Huaiming Guo, Physica C 436 (2006) 14.

19 P. W. Anderson, in Frontiers and Borderlines in Many Particle Physics, edited by R. A. Broglia and J. R. Schrieffer (North-Holland, Amsterdam, 1987), p. 1; Science 235 (1987) 1196.

20 Shiping Feng, Jihong Qin, and Tianxing Ma, J. Phys.: Condens. Matter 16 (2004) 343.

21 See, e.g., the review, Shiping Feng, Huaiming Guo, Yu Lan, and Li Cheng, Int. J. Mod. Phys. B 22 (2008) 3757.

22 See, e.g., the review, S. Hüfner, M. A. Hossain, A. Damascelli, and G. A. Sawatzky, Rep. Prog. Phys. 71 (2008) 062501 .

23 Huaiming Guo and Shiping Feng, Phys. Lett. A 361 (2007) 382.

${ }^{24}$ I. M. Vishik, W. S. Lee, R. H. He, M. Hashimoto, Z. Hussain, T. P. Devereaux, and Z.-X. Shen, New J. Phys. 12 (2010) 105008. 